Provided for non-commercial research and education use. Not for reproduction, distribution or commercial use.

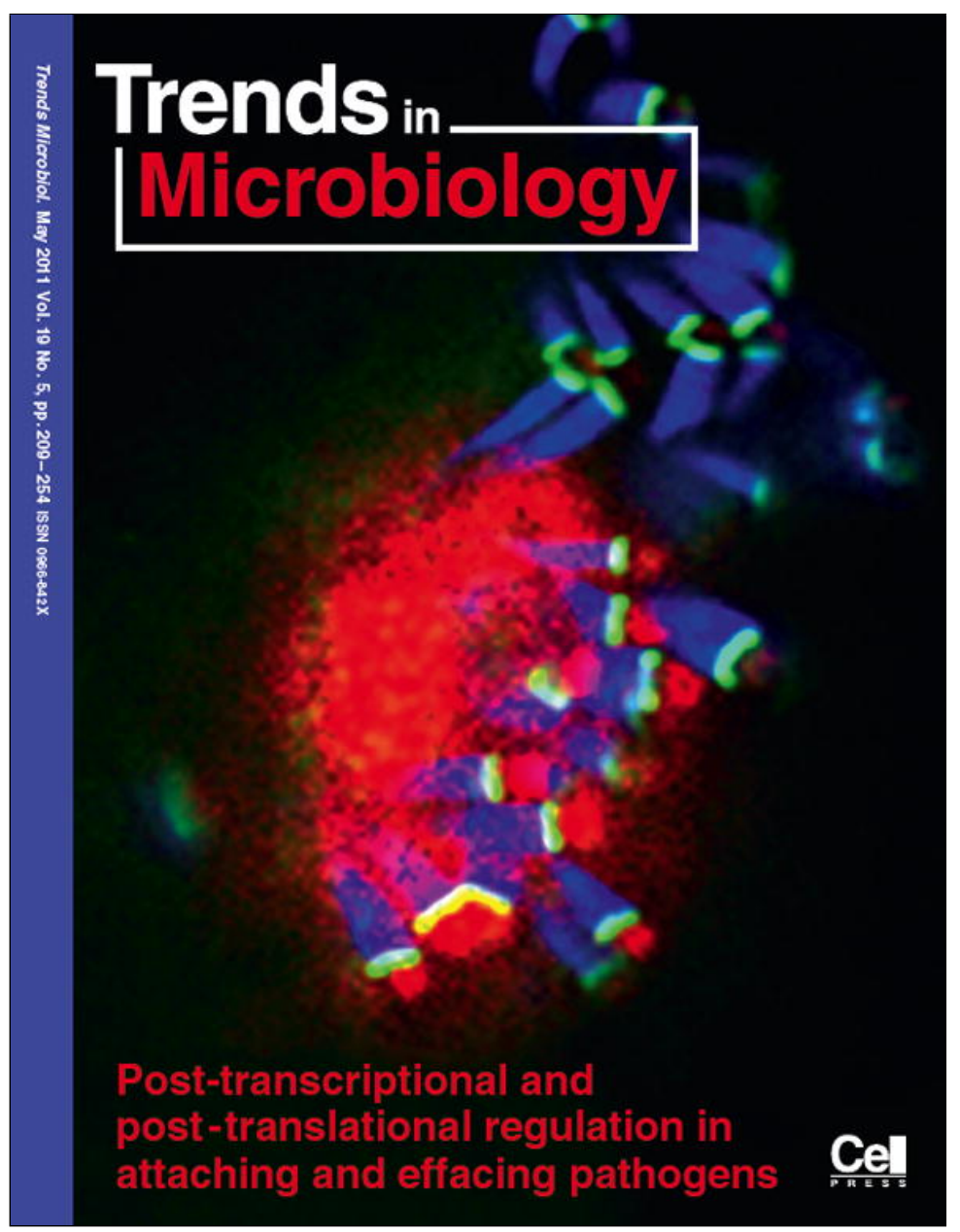

This article appeared in a journal published by Elsevier. The attached copy is furnished to the author for internal non-commercial research and education use, including for instruction at the authors institution and sharing with colleagues.

Other uses, including reproduction and distribution, or selling or licensing copies, or posting to personal, institutional or third party websites are prohibited.

In most cases authors are permitted to post their version of the article (e.g. in Word or Tex form) to their personal website or institutional repository. Authors requiring further information regarding Elsevier's archiving and manuscript policies are encouraged to visit:

http://www.elsevier.com/copyright 


\title{
Adherence and biofilm formation of non-Candida albicans Candida species
}

\author{
Sónia Silva ${ }^{1}$, Melyssa Negri ${ }^{1}$, Mariana Henriques ${ }^{1}$, Rosário Oliveira ${ }^{1}$, \\ David W. Williams ${ }^{2}$ and Joana Azeredo ${ }^{1}$ \\ ${ }^{1}$ Institute for Biotechnology and Bioengineering, Universidade do Minho, Campus de Gualtar 4710-057, Braga, Portugal \\ ${ }^{2}$ Tissue Engineering and Reparative Dentistry, School of Dentistry, Heath Park, Cardiff CF14 4XY, UK
}

\begin{abstract}
Most cases of candidosis have been attributed to Candida albicans, but recently non-C. albicans Candida species have been identified as frequent human pathogens. Candida pathogenicity has been attributed to several factors, including adhesion to medical devices and/or host cells, biofilm formation, and secretion of hydrolytic enzymes (proteases, phospholipases and haemolysins). Although 'new' Candida species are emerging, there is still a lack of information about their pathogenicity. This review discusses recent advances in our knowledge of Candida glabrata, Candida parapsilosis and Candida tropicalis virulence factors, specifically those of adhesion and biofilm formation, which are key components in Candida pathogenicity.
\end{abstract}

\section{Emergence of non-Candida albicans Candida species} Twenty years ago Candida albicans accounted for 70-80\% of the Candida isolates recovered from infected patients [1]. Despite the majority of candidosis cases being attributed to $C$. albicans, an increasing number of infections caused by non-C. albicans Candida (NCAC) species, specifically Candida glabrata, Candida parapsilosis and Candida tropicalis, have been reported over the past two decades [2-4].

For many years, $C$. glabrata was considered a relatively non-pathogenic saprophyte of the normal flora of healthy humans, and was not readily associated with serious infection. However, C. glabrata can rapidly disseminate throughout the body, and infection with this species is associated with a high mortality rate. Moreover, C. glabrata is of added concern because of its inherent resistance to certain antifungal agents [1]. C. parapsilosis is generally regarded as one of the least virulent yeast species, although it is now a frequent cause of candidaemia, commonly related to poor hand hygiene of healthcare workers [2]. C. tropicalis has emerged as the second or third most common agent of candidaemia, mainly in oncology patients, and is often associated with nosocomial urinary-tract infections [4].

The apparently increasing involvement of NCAC species in human candidosis could be related in part to improvements in diagnostic methods, such as the use of primary agars with the ability to differentiate between Candida species, and the introduction of molecular diagnostic techniques into routine use [5]. A number of other

Corresponding author: Henriques, M. (mcrh@deb.uminho.pt). factors have been implicated in the increasing prevalence of Candida species, including the introduction and more widespread use of certain medical practices, such as immunosuppressive therapy, the use of broad-spectrum antibiotics, and an increase in the number of invasive surgical procedures, such as organ transplantations [6,7]. Furthermore, the increasing number of Candida species in candidosis could also be a reflection of species selection in the presence of certain antifungals, given the higher level of resistance demonstrated by several NCAC species [8].

The pathogenesis of invasive candidosis is facilitated by a number of factors, including the ability to adhere to medical devices and/or host cells and to form biofilms. It is also important to highlight the ability of some Candida species to switch from yeast to filamentous growth forms, with the latter thought to increase the ability of the organism to invade host tissues. C. albicans is a true polymorphic organism, able to grow as hyphae and/or pseudohyphae, and as blastospores (yeast). C. tropicalis produces oval blastospores and pseudohyphae, and, according to some reports, true hyphae. In the case of $C$. parapsilosis, blastospore growth is prevalent, and although this species does not produce true hyphae, it can on occasion generate pseudohyphae. By contrast, C. glabrata is not polymorphic, growing only as blastospores [9].

Despite significant research aimed at identifying the pathogenic factors of fungi, particularly in C. albicans, relatively little is known about the virulence determinants of NCAC species. This review aims to provide a contemporary overview of the virulence factors associated with three of these clinically important NCAC species (C. glabrata, C. parapsilosis and C. tropicalis), with particular focus given to aspects of their adhesion and biofilm formation.

\section{Adhesion ability of NCAC species}

The first event in Candida infection is its adherence of the organism to host and/or medical-device surfaces, often leading to the formation of biofilms [10]. Thus, adhesion is an extremely important step in the infection process, and the extent of adhesion is dependent on microbial, host and abiotic surface properties, such as cell-surface hydrophobicity and cell-wall composition [11,12].

The yeast cell wall is the site for physicochemical interactions between the microorganism and host surfaces, leading to its adherence. The cell surface of $C$. glabrata reportedly exhibits a degree of hydrophobicity comparable 
with that of $C$. albicans; however, these results were obtained in a study of a limited number of C. glabrata strains [13]. Interestingly, whereas the hydrophobicity of C. albicans appears to be extremely sensitive to specific growth conditions, the cell-surface hydrophobicity of numerous isolates of $C$. glabrata was found to be comparatively stable under those same growth conditions [14]. In addition, Camacho et al. [15] did not find a correlation between the level of hydrophobicity and adherence of $C$. albicans, C. tropicalis and C. parapsilosis to siliconized latex catheters, demonstrating that cell hydrophobicity alone was not a predictor of adhesion. However, Panagoda et al. [16] demonstrated that the initial adhesion of $C$. parapsilosis was associated with the hydrophobicity of acrylic surfaces. Recently Silva et al. [17] showed that $C$. glabrata adhered to silicone in the presence of urine at higher levels than did either C. tropicalis or C. parapsilosis. Additionally, C. glabrata has also been reported to have a two-fold greater tendency to adhere to dentureacrylic surfaces compared with $C$. albicans [18]. Furthermore, $C$. glabrata has been shown to have higher adherence to urinary epithelial cells than do other NCAC species [19]. C. tropicalis has the ability to colonize urinary epithelial cells [20]; however, the extent of adhesion is straindependent. In terms of epithelial and endothelial adhesion ability, few studies have been undertaken with $C$. parapsilosis.

An important factor that has correlated with the adhesion ability of Candida species is the presence of specific cell-wall proteins, often referred to as adhesins. Groot et al. [21] identified 23 C. glabrata cell-wall adhesins, which were deemed to be involved in adherence to human epithelia and in biofilm formation. A major group of adhesins of C. glabrata is encoded by the EPA gene family [21-23]. The ability of $C$. glabrata to adhere to different biomaterials and epithelia could be a reflection of epithelial adhesin (EPA) expression on the surface of cells, which is induced by the presence of nicotinic acid [24]. Although there are relatively few studies concerning $C$. glabrata Epa adhesins, it is known that Epa1p is a $\mathrm{Ca}^{2+}$-dependent lectin [25]. Furthermore, despite the large number of $E P A$ genes, it has been shown that deletion of EPA1 alone reduces adherence in vitro [25]. Furthermore, although EPA6 is not expressed in vitro, its expression increases during in vivo urinary infection, suggesting that $C$. glabrata can adapt with environmental conditions to enhance its adherence [26]. A bioinformatics search of pathogenspecific gene families of Candida species revealed a number of genes encoding cell-wall proteins in C. parapsilosis. This study included five genes for agglutinin-like sequence (ALS) proteins and six genes for predicted glycophosphatidylinositol-anchored protein 30 (Pga30) [27]. Unfortunately, there has been no further work in studying the role played by these proteins in $C$. parapsilosis adhesion. Concerning proteins of the $C$. tropicalis cell wall, at least three ALS-encoding genes have been identified [28], but to our knowledge, no further work has been undertaken in this area.

The pathogenesis of mucosal candidosis has mainly been investigated using animal models, but recently, reconstituted human epithelium (RHE) was successfully used to study in vitro invasion by Candida species. In this (a)

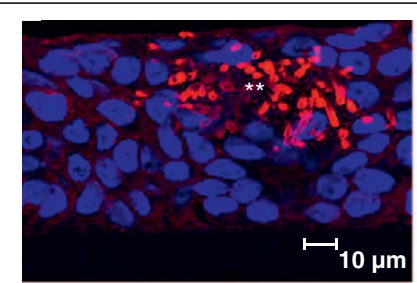

Candida parapsilosis

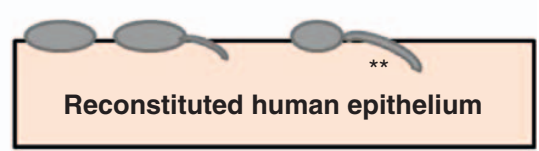

(b)

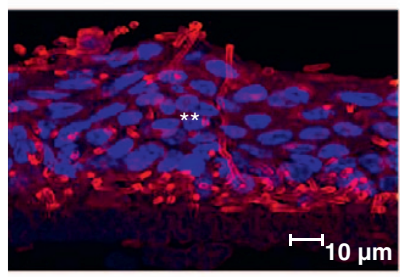

Candida tropicalis

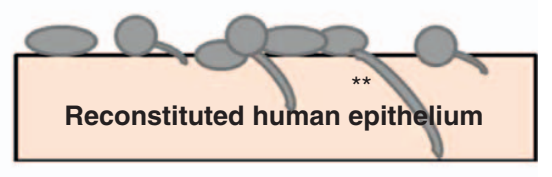

(c)

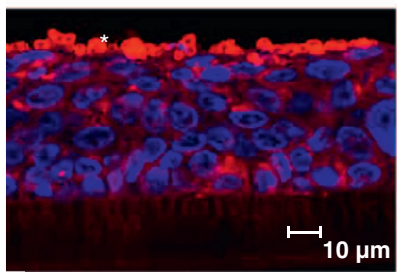

Candida glabrata

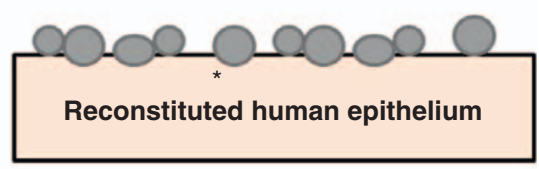

TRENDS in Microbiology

Figure 1. Confocal laser scanning microscopy images and scheme of Candida species infecting of a reconstituted human oral epithelium after $12 \mathrm{~h}$ of colonization. Candida yeast and filaments are shown in red, and the nucleic of the epithelial cells are depicted in blue. (a) $C$. parapsilosis showing moderate colonization, and invasion; (b) $C$. tropicalis showing extensive colonization and invasion; (c) C. glabrata showing colonization but no invasion. *Yeast; ** filamentous forms. 
model, C. parapsilosis exhibited a high ability to colonize the RHE, despite a lower ability to invade this tissue [29,30] (Figure 1a). Furthermore, Gácser et al. [31] showed that $C$. orthopsilosis caused similar damage to RHE, whereas C. metaparapsilosis had comparatively reduced virulence. Jayatilake et al. [32] showed increased ability of C. tropicalis (Figure 1b) to colonize and invade RHE, similarly to $C$. albicans, compared with C. glabrata (Figure 1c) and C. parapsilosis (Figure 1b). C. glabrata was shown to be non-invasive in this model [32]. Therefore, given the inability of C. glabrata to generate filamentous forms, it might be concluded that the filamentous form is important for host tissue invasion.

\section{Hydrolytic enzyme secretion by NCAC species}

Candida species are able to produce and secrete several hydrolytic enzymes, including proteases, phospholipases (PLs) and haemolysins. The activity of these enzymes has been associated with candidal adhesion, cell damage and invasion of host tissue.

\section{Secreted aspartyl proteinases}

The secretion of aspartyl proteinases (Sap1 to Sap10) by $C$. albicans is recognized as an important virulence determinant for this species [33]. Saps facilitate colonization and invasion of host tissues through the disruption of host mucosal membranes [34], and by degrading important immunological and structural defence proteins [35].

With regard to C. glabrata, only one study has shown that this species is capable of secreted proteinase production, but the type of proteinase was not specified [36]. In addition, compared with C. albicans, C. parapsilosis has been shown to possess relatively low Sap activity [37]. Only three $S A P$ genes have been identified in C. parapsilosis (SAPP1-3), two of which remain largely uncharacterized [38]. It has been reported that the expression of $S A P P 1-3$ genes varies with the clinical isolate of $C$. parapsilosis when grown in contact with an oral epithelium and even in planktonic form [30]. However, an associated trend was also reported, which related Sap production and the site of isolation of the organism; both vaginal and skin isolates of $C$. parapsilosis exhibited higher in vitro Sap activity than blood isolates [39]. C. parapsilosis poorly invades oral epithelium, but can nevertheless induce significant damage, which was related to specific $S A P$ gene expression [30].

As with $C$. albicans, in vitro studies revealed that $C$. tropicalis secretes high levels of Saps in culture media containing bovine serum albumin as the sole source of nitrogen. Furthermore, $C$. tropicalis possesses at least four genes encoding Saps (SAPT1-4) [40,41], although Sapt1p is the only one that has been purified from culture supernatant, and biochemically characterized and crystallized $[42,43]$. Sap secretion by $C$. tropicalis has also been reported on the surface of fungal elements penetrating tissues during disseminated infection, and on macrophages following phagocytosis of yeast cells [34]. Sap expression by $C$. tropicalis in the oral environment is not associated with invasion or tissue damage [44], a finding similar to that reported for C. albicans $[45,46]$.

Despite the high number of studies concerning the role of Saps on C. albicans virulence, little emphasis has been given to NCAC species Saps. Moreover it is important to highlight that although several studies have highlighted the differential expression and potential roles of various $S A P$ genes during colonization and infection of host by Candida species, there were discrepancies in the results obtained. The reasons for such discrepancies could relate to differences in the sensitivities of the methods used, intrinsic differences even in apparently similar infection models (e.g. RHE and murine), and interspecies and strain variability $[45,47]$.

\section{PLs and lipases}

In addition to Saps, enzymes categorized as lipases and PLs are often considered to be factors associated with Candida pathogenicity.

PLs are enzymes that hydrolyze phospholipids to fatty acids. Depending on the different and specific ester bonds cleaved, these enzymes have been classified into PLs A, B, $\mathrm{C}$ and D [48]. The production of all classes of PLs has been described for Candida species, and their presence could conceivably contribute to host cell-membrane damage, which could promote cell damage and/or expose receptors to facilitate adherence of Candida [49].

Several studies have indicated that NCAC species are able to produce extracellular PLs [50,51], albeit at lower levels compared with $C$. albicans [45]. There have been a number of contradictory findings, with some investigators reporting PL activity in $51 \%$ of strains assayed, whereas others have failed to detect PL activity in assayed strains [45]. Although C. tropicalis appears to have a reduced ability to produce extracellular PLs, this is strongly strain-dependent $[21,38,52,53]$. Furthermore, although a few studies of PL activity have been undertaken for $C$. tropicalis and $C$. parapsilosis, none has been reported for C. glabrata [54].

Lipases are involved both in the hydrolysis and synthesis of triacylglycerols. These enzymes are stable at high temperatures and in organic solvents and are resistant to proteolysis. In C. albicans, 10 lipase genes have been identified [55]. Gácser et al. [56] showed that C. albicans mutants deficient in CaLIP8 were significantly less virulent in a murine intravenous infection model. This work clearly indicated that Lip8p was likely to be an important virulence factor for this particular species. For $C$. parapsilosis, CpLIP1 and CpLIP2 have been reported, with the latter known to encode for an active protein [57,58]. Recently, Gácser et al. [31] demonstrated that a lipase inhibitor significantly reduced tissue damage during $C$. parapsilosis infection of reconstituted human tissues, and that $C p L I P 1 / C p L I P 2$ mutants formed thinner and less complex biofilms. Recent genomic DNA sequencing suggests that two additional $C p L I P$ genes might exist in $C$. parapsilosis [2]. Sequences similar to C. albicans (LIP110) were also detected in $C$. tropicalis, but not in $C$. glabrata [59]. However, no studies have been performed to investigate the role of these genes in the virulence of $C$. tropicalis.

\section{Haemolytic activity}

Pathogenic microorganisms can grow in the host by using haemin or haemoglobin as a source of iron. Haemolysins 
Table 1. C. tropicalis, C. parapsilosis and C. glabrata biofilm characteristics

\begin{tabular}{|l|l|l|l|l}
\hline Species & Biofilm formation & Matrix composition & Biofilm structure & Discontinuous compact monolayer \\
\hline C. tropicalis & + & Low level of carbohydrates and proteins & Discontinuous monolayer or multilayer \\
\hline C. parapsilosis & + & High level of carbohydrates and low levels of proteins & Compact monolayer or multilayer \\
\hline C. glabrata & +- & High level of carbohydrates and proteins &
\end{tabular}

${ }^{a}+$, Robust biofilm growth; +/-, less biofilm growth.

are produced by Candida species to degrade haemoglobin and extract elemental iron from host cells. Thus, haemolysins are likely to be key virulence factors as might promote pathogen survival and persistence in the host $[60,61]$. C. albicans has the ability to further utilize the acquired iron to produce a haemolytic factor that can release more haemoglobin by lysing erythrocytes $[60,61]$. Candida haemolytic activity can be concentrated by absorption on a concanavalin-A sepharose matrix. Analysis of the absorbed haemolysin demonstrated that the haemolytic factor involved was mannoprotein in origin [61]. Production of this haemolysin appears to be regulated by the presence of glucose in the growth medium. C. glabrata, $C$. parapsilosis and $C$. tropicalis are also able to produce haemolysins in vitro and at various levels, inducing partial or total erythrocyte lysis [59]. The genetic expression of haemolytic activity by Candida is, at present, poorly understood, but Luo et al. [62] showed that the haemolysinlike protein $(H L P)$ gene was associated with the hemolytic activity of $C$. glabrata. Clearly, investigations are at an early stage with respect to the haemolysin activity of Candida species. This is highlighted by the fact that for pathogenic fungi, cloning, disruption and virulence evaluation has only led to the identification of the C. albicans haemolysin. Further investigations aimed at determining the role of haemolysins in the virulence of different NCAC species are required to establish whether these molecules are universal virulence factors of Candida species.

Apart from C. albicans, our knowledge of fungal adhesion, colonization and invasion of human epithelia and medical devices remains limited. The first contact between microorganisms and host surfaces is generally via the fungal cell wall, and this is therefore believed to play a key role in the pathogenicity of Candida species. Therefore, elucidation of the physicochemical properties (e.g. hydrophobicity), including chemical composition, should facilitate better understanding of the pathogenesis of NCAC infections. Furthermore, establishing the role of cell-wall components in the infection process could also enhance management strategies by potentially identifying appropriate and alternative targets for antifungal drugs.

\section{Biofilm formation by NCAC species}

Initial attachment of Candida to the host or/and medical devices is followed by cell division, proliferation and subsequent biofilm development [63]. Biofilms are surfaceassociated communities of microorganisms embedded within an extracellular matrix (ECM), and are considered the most prevalent growth form of microorganisms [64]. Biofilm formation is a potent virulence factor for a number of Candida species, as it confers significant tolerance to antifungal therapy, primarily by limiting the penetration of substances through the biofilm matrix. Growth as a biofilm also serves to protect the embedded cells from host immune responses [65]. Moreover, C. albicans, C. parapsilosis, C. tropicalis and C. glabrata isolates are adept at forming biofilms, and their presence during infection has been linked to higher mortality rates compared with isolates incapable of forming biofilms [66]. It is thought that the formation of mature biofilms and subsequent production of the ECM is strongly dependent upon species, strain, and environmental conditions such as $\mathrm{pH}$, medium composition and oxygen [63,67].

Table 1 summarizes the most relevant characteristics of C. parapsilosis, C. tropicalis and C. glabrata biofilms. C. albicans biofilm formation is associated with the dimorphic switch between yeast and hyphal growth, and biofilms of this species generally have two distinct layers: a thin, basal yeast layer and a thicker, less compact hyphal layer [68]. In contrast to C. albicans, C. parapsilosis biofilms (Figure 2) tend to be thinner, less structured, and consist almost exclusively of aggregated blastospores [69]. Interestingly, biofilm formation by $C$. parapsilosis is strongly straindependent [64]. The selective preference of this species for plastic medical devices is of particular interest, as biofilm formation enhances the capacity of $C$. parapsilosis
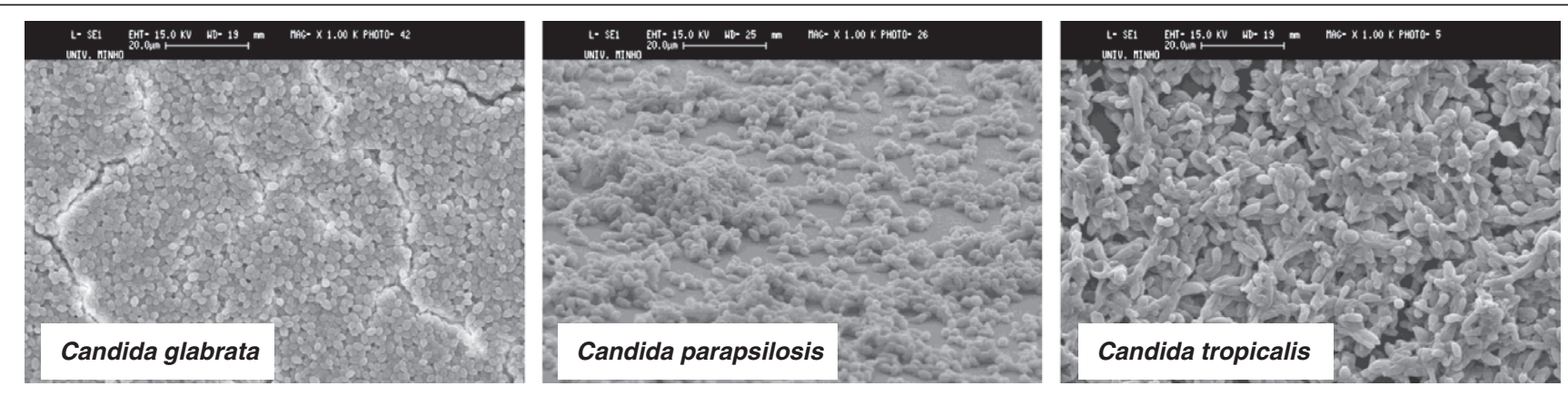

$\overline{\text { TRENDS in Microbiology }}$

Figure 2. Biofilm structure of C. glabrata, C. parapsilosis and C. tropicalis. Scanning electron microscopy images taken after biofilm growth in SDB after $48 \mathrm{~h}$. 
to colonize catheters and intravascular central lines [2]. $C$. tropicalis clinical isolates have been classified as being prolific biofilm formers $[21,64]$, and their mature biofilms consist of a dense network of yeast cells in addition to evident filamentous morphologies (Figure 2) [64]. A genetic screen of $C$. glabrata biofilm mutants identified four genes involved in biofilm formation, and biofilm growth conditions induced the transcription of EPA6 and EPA7 [70]. C. glabrata biofilms exhibit a more compact structure than those of $C$. tropicalis and $C$. parapsilosis biofilms (Figure 2). Additionally, two newly identified Candida species (C. orthopsilosis and C. metapsilosis) have also been shown to be capable of forming biofilms [71]. It must be stressed that comparisons of the biofilms formed by $C$. glabrata, C. tropicalis and C. parapsilosis should always consider the inherent physiological differences between these species, such as relative cell size, morphology and biochemistry. When comparing Candida biofilms, future research should carefully define the most appropriate parameters to investigate.

Biofilm formation and matrix composition are strongly dependent on the strain and the environmental conditions (medium composition, $\mathrm{pH}$ and oxygen) used [63]. Recently, Silva et al. [17] showed that $C$. glabrata produced higher biofilm biomass on silicone in the presence of urine, compared with C. parapsilosis and C. tropicalis. Conversely, $C$. tropicalis and $C$. parapsilosis yielded a higher biofilm biomass in Sabouraud dextrose broth (SDB) compared with C. glabrata [64]. These results are in accordance with Shin et al. [72], who reported that biofilm formation by $C$. glabrata was lower than that of other NCAC species, when cultured in nutritionally rich media. C. glabrata cells are smaller $(1-4 \mu \mathrm{m})$ than those of $C$. tropicalis $(4-8 \mu \mathrm{m})$ and C. parapsilosis $(2.5-4 \mu \mathrm{m})$ and also have a narrower spectrum of carbohydrate utilization. Unlike C. parapsilosis and C. tropicalis, C. glabrata is unable to generate filamentous forms, which might also be expected to exhibit different metabolic activities, and thus this might contribute to the lower amount of C. glabrata biomass in rich medium. Such factors undoubtedly reflect the inherent physiological differences between these species, and could have significance for their pathogenic potential. Biofilms are readily formed by $C$. parapsilosis cells grown in media containing high glucose and lipid concentrations, which can be thought of being analogous to bloodstream infections in patients receiving parenteral nutrition. These results highlight the observation that biofilm formation and consequent matrix production are strongly dependent on the source of nutrients available.

The biofilm matrix of $C$. albicans is mainly composed of carbohydrates, proteins, phosphorus and hexosamines [73]. The matrix of C. glabrata biofilms produced in SDB has been found to contain high levels of proteins and carbohydrates [64]. The ECM of C. parapsilosis biofilms is composed of large quantities of carbohydrates, and the protein content is low compared with biofilms of C. glabrata and C. tropicalis [64]. The matrix of C. tropicalis biofilms also has a notable difference from those of the other NCAC species. Although the $C$. tropicalis matrix contains carbohydrates, proteins, hexosamine, phosphorus and uronic acid [74], it has lower amounts of carbohydrates and protein [64]. Furthermore,

\section{Box 1. Outstanding questions}

- Is the emergence of NCAC species related to the development of molecular diagnostic approaches, or to changes in their virulence determinants?

- What determines the switch from harmless commensal into infectious pathogen for NCAC species?

- What are the genes and proteins expressed or regulated during infection with NCAC species?

the major component in the C. tropicalis biofilm matrix is hexosamine (27\%). In addition, $C$. tropicalis biofilms can be partially detached after treatment with lipase type VII and chitinase, whereas biofilms of $C$. albicans are detachable after treatment with proteinase $\mathrm{K}$, chitinase, DNase I or $\beta$ $\mathrm{N}$-acetylglucosamidase [74]. These findings highlight that detachment and destruction of NCAC biofilms is dependent on matrix composition. This opens the possibility that some medical devices could be coated with specific types of hydrolytic enzymes as a means of preventing biofilm formation by NCAC species.

Several genes and proteins have been reported as essential for Candida biofilm formation and matrix composition. Two recent studies showed that $C$. parapsilosis LIP knockout mutants had a decreased ability to form biofilms. C. parapsilosis lipase mutants produce significantly less biofilm than wild-type strains [31], and the biofilm and cell wall regulator $(B C R)$ gene was found to be necessary for proper biofilm formation [75]. Notably, the biofilm-deficient $C$. parapsilosis lipase mutants were less virulent in tissue culture infection models and also in mice [31]. Little is known about the genes involved in biofilm formation for C. glabrata and C. tropicalis.

The studies described above highlight the diversity found for C. glabrata, C. parapsilosis and C. tropicalis, in terms of biofilm-forming ability, structure and matrix composition. It must be emphasized that it is clearly very important to continue these studies to elucidate the inherent differences between these and other NCAC species to identify and combat their involvement in infection.

\section{Concluding remarks}

An alteration to host immunity is generally required by opportunistic pathogens, such as Candida, to switch from harmless commensal microorganisms to potentially lifethreatening human pathogens. Candida utilizes several genes, which play an important role in adhesion, biofilm formation and secretion of enzymes, and are consequently involved in virulence. Given these findings and the increased incidence of candidosis caused by NCAC species, especially C. glabrata, C. parapsilosis and C. tropicalis, and the unacceptably high morbidity and mortality associated with them, it is essential to increase our knowledge about the factors associated with adhesion and biofilm formation in NCAC infections. Further studies in this area will also contribute towards the identification of new targets for future therapeutics against these recently emerged pathogens (Box 1).

\section{Acknowledgments}

This work was supported by the Fundação para Ciência e Tecnologia (FCT), Portugal (grant SFRH/BD/28341/2006 to S.S.). and the 
Coordenação de Aperfeiçoamento de Pessoal de Nível Superior (CAPES), Brazil (grant BEX-4642/06-6 to M.N.).

\section{References}

1 Fidel, P.L., Jr et al. (1999) Candida glabrata: review of epidemiology, pathogenesis, and clinical disease with comparison to C. albicans. Clin. Microbiol. Rev. 12, 80-96

2 Trofa, D. et al. (2008) Candida parapsilosis, an emerging fungal pathogen. Clin. Microbiol. Rev. 21, 606-662

3 Pfaller, M.A. and Diekema, D.J. (2007) Epidemiology of invasive candidiasis: a persistent public health problem. Clin. Microbiol. Rev 20, 133-163

4 Kothavade, R.J. et al. (2010) Candida tropicalis: its prevalence, pathogenicity and increasing resistance to fluconazole. J. Med. Microbiol. 59, 873-880

5 Liguori, G. et al. (2009) Oral candidiasis: a comparison between conventional methods and multiplex polymerase chain reaction for species identification. Oral Microbiol. Immunol. 24, 76-78

6 Kotloff, R.M. et al. (2004) Pulmonary complications of solid organ and hematopoietic stem cell transplantation. Am. J. Resp. Crit. Car. Med. $170,22-48$

7 Kojic, E.M. and Darouichee, R.O. (2004) Candida infections of medical devices. Clin. Microbiol. Rev. 17, 255-267

8 Gonzalez, G.H. et al. (2007) Oral Candidiasis in children and adolescents with cancer. Identification of Candida spp. Med. Oral Patol. Oral Cir. Bucal. 12, 419-423

9 Calderone, R.A. (2002) Introduction and historical perspectives. In Candida and candidiasis (Calderone, R., ed.), pp. 15-25, Washington D.C, ASM Press

10 Chandra, J. et al. (2001) Biofilm formation by the fungal pathogen Candida albicans: development, architecture, and drug resistance. $J$. Bacteriol. 183, 5385-5394

11 Henriques, M. et al. (2002) Experimental methodology to quantify Candida albicans cell surface hydrophobicity. Biotechnol. Lett. 24, 1111-1115

12 Chaffin, W.L. (2008) Candida albicans cell wall proteins. Microbiol. Mol. Biol. Rev. 72, 495-544

13 Hazen, K.C. et al. (1986) Influence of growth conditions on cell surface hydrophobicity of Candida albicans and Candida glabrata. Infect. Immun. 54, 269-271

14 Kikutani, H. and Makino, S. (1992) The murine autoimmune diabetes model: NOD and related strains. Adv. Immunol. 51, 285-322

15 Camacho, D. et al. (2007) The effect of chlorhexidine and gentian violet on the adherence of Candida spp. to urinary catheters. Mycopathologia 163, 261-266

16 Panagoda, G.J. et al. (2001) Adhesion of Candida parapsilosis to epithelial and acrylic surfaces correlates with cell surface hydrophobicity. Mycoses 44, 29-35

17 Silva, S. et al. (2010) Silicone colonisation by non-Candida albicans Candida species in the presence of urine. J. Med. Microbiol. 59, 747-754

18 Luo, G. and Samaranayake, L.P. (2002) Candida glabrata, an emerging fungal pathogen, exhibits superior relative cell surface hydrophobicity and adhesion to denture acrylic surfaces compared with Candida albicans. APMIS 110, 601-610

19 Negri, M. et al. (2010) Crystal violet expedite method for quantification adhesion to epithelial cells. Br. J. Microbiol. 67, 120-125

20 Negri, M. et al. (2010) Examination of potential virulence factors of Candida tropicalis clinical isolates from hospitalized patients. Mycopathologia 169, 175-182

21 Groot, P.W.J. et al. (2008) The cell wall of the human pathogen Candida glabrata: differential incorporation of novel adhesin-like wall proteins. Eukaryotic Cell 7, 1951-1964

22 De Las Penas, A. et al. (2003) Virulence-related surface glycoproteins in the yeast pathogen Candida glabrata are encoded in subtelomeric clusters and subject to RAP1- and SIR-dependent transcriptional silencing. Genes Dev. 17, 2245-2258

23 Zupancic, M.L. et al. (2008) Glycan microarray analysis of Candida glabrata adhesin ligand specificity. Mol. Microb. 68, 547-559

$24 \mathrm{Ma}$, B. et al. (2007) Assimilation of NAD+ precursors in Candida glabrata. Mol. Microb. 66, 14-25

25 Cormack, B.P. et al. (1999) An adhesin of the yeast pathogen Candida glabrata mediating adherence to human epithelial cells. Science 285, $578-582$
26 Domergue, R. et al. (2005) Nicotinic acid limitation regulates silencing of Candida adhesins during UTI. Science 308, 866-870

27 Butler, G. et al. (2009) Evolution of pathogenicity and sexual reproduction in eight Candida genomes. Nature 459, 657-662

28 Hoyer, L.L. et al. (2001) Characterization of agglutinin-like sequence genes from non-albicans Candida and phylogenetic analysis of the ALS family. Genetics 157, 1555-1567

29 Schaller, M. et al. (2006) Models of oral and vaginal candidiasis based on in vitro reconstituted human epithelia. Nat. Protoc. 1, 2767-2773

30 Silva, S. et al. (2009) Characterization of Candida parapsilosis infection of an in vitro reconstituted human oral epithelium. Eur. J. Oral Sci. 117, 669-675

31 Gácser, A. et al. (2007) Virulence of Candida parapsilosis, Candida orthopsilosis, and Candida metapsilosis in reconstituted human tissue models. Fungal Genet. Biol. 44, 1336-1341

32 Jayatilake et al. (2006) Quantitative evaluation of tissue invasion by wild type, hyphal and SAP mutants of Candida albicans, and nonalbicans Candida species in reconstituted human oral epithelium. J. Oral Pathol. Med. 35, 481-491

33 Monod, M. et al. (2002) Secreted proteases from pathogenic fungi. Int. J. Med. Microbiol. 292, 405-419

34 Ruchel, R. et al. (2004) Candida acid proteases. J. Med. Vet. Mycol. 30 (Suppl. 1), 123-132

35 Pichova, I. et al. (2001) Secreted aspartic proteases of Candida albicans, Candida tropicalis, Candida parapsilosis and Candida lusitaniae. Inhibition with peptidomimetic inhibitors. Eur. J. Biochem. 268, 2669-2677

36 Chakrabarti, A. et al. (1991) In vitro proteinase production by Candida species. Mycopathologia 114, 163-168

37 Kobayashi, C. et al. (2004) Candiduria in hospital patients: a study prospective. Mycopathologia 158, 49-52

38 Merkerova, M. et al. (2006) Cloning and characterization of Sapp2p, the second aspartic proteinase isoenzyme from Candida parapsilosis. FEMS Yeast Res. 6, 1018-1026

39 Dagdeviren, M. et al. (2005) Acid proteinase, phospholipase and adherence properties of Candida parapsilosis strains isolated from clinical specimens of hospitalised patients. Mycoses 48, 321-326

40 Zaugg, C. et al. (2001) Secreted aspartic proteinase family of Candida tropicalis. Infect. Immun. 69, 405-412

41 Togni, G. et al. (1991) Isolation and nucleotide sequence of the extracellular acid protease gene (ACP) from the yeast Candida tropicalis. FEBS Lett. 286, 181-185

42 Symersky, J. et al. (1997) High-resolution structure of the extracellular apartic proteinase from Candida tropicalis yeast. Biochemistry 36, 12700-12710

43 Borg, M. and Ruchel, R. (1990) Demonstrating of fungal proteinase during pahocytosis of Candida albicans and Candida tropicalis. J. Med. Vet. Mycol. 28, 3-14

44 Silva, S. et al. (2011) The role of secreted aspartyl proteinases in Candida tropicalis invasion and damage of oral mucosa. Clin. Microbiol. Infect. 17, 264-272

45 Lermann, U. and Morschhauser, J. (2008) Secreted aspartic proteases are not required for invasion of reconstituted human epithelia by Candida albicans. Microbiology 154, 3281-3295

46 Naglik, J.R. et al. (2008) Quantitative expression of the Candida albicans secreted aspartyl proteinase gene family in human ora and vaginal candidiasis. Microbiology 154, 3266-3280

47 Correia, A. et al. (2010) Limited role of secreted aspartyl proteinases Sap1 to Sap6 in Candida albicans virulence and host immune response in murine hematogenously disseminated candidiasis. Infect. Immun. $78,4839-4849$

48 Mukherjee, P.K. et al. (2001) Reintroduction of the PLB1 gene into Candida albicans restores virulence in vivo. Microbiology 147, 2585-2597

49 Ghannoum, M.A. (2000) Potential role of phospholipases in virulence and fungal pathogenesis. Clin. Microbiol. Rev. 13, 122-143

50 Galan-Ladero, M.A. et al. (2010) Enzymatic activities of Candida tropicalis isolated from hospitalized patients. Med. Mycol. 48, 207-210

51 Cafarchia, C. et al. (2008) Phospholipase activity of yeasts from wild birds and possible implications for human disease. Med. Mycol. 46, $429-434$

52 Furlaneto-Maia, L. et al. (2007) In vitro evaluation of putative virulence attributes of oral isolates of Candida spp. obtained from elderly healthy individuals. Mycopathologia 166, 209-217 
53 Dostal, J. et al. (2005) The precusor of secreted aspartic proteinase Sapp1p from Candida parapsilosis can be activated both autocatalytically and by membrane bound processing proteinase. Biol. Chem. 386, 791-799

54 Hube, B. et al. (2001) The role and relevance of phospholipase D1 during growth and dimorphism of Candida albicans. Microbiology 147, 879-889

55 Gácser, A. et al. (2007) Lipase 8 affects the pathogenesis of Candida albicans. Infect. Immun. 75, 4710-4718

56 Brunel, L. et al. (2004) High-level expression of Candida parapsilosis lipase/acyltransferase in Pichia pastoris. J. Biotechnol. 111, 41-50

57 Neugnot, V. et al. (2002) The lipase/acyltransferase from Candida parapsilosis: molecular cloning and characterization of purified recombinant enzymes. Eur. J. Biochem. 269, 1734-1745

58 Filler, S.G. et al. (1991) Candida albicans stimulates endothelial cell eicosanoid production. J. Infect. Dis. 164, 928-935

59 Luo, G. et al. (2001) Candida species exhibit differential in vitro hemolytic activities. J. Clin. Microbiol. 39, 2971-2974

60 Watanabe, T. et al. (1999) Characterization of a haemolytic factor from Candida albicans. Microbiology 145, 689-694

61 Manns, J.M. et al. (1994) Production of a hemolytic factor by Candida albicans. Infect. Immun. 62, 5154-5156

62 Luo, G. et al. (2004) Reverse transcriptase polymerase chain reaction (RT-PCR) detection of HLP gene expression in Candida glabrata and its possible role in in vitro haemolysin production. APMIS 112, 283290

63 Ramage, G. et al. (2006) Candida biofilms on implanted biomaterials: a clinically significant problem. FEMS Yeast Res. 6, 979-986

64 Silva, S. et al. (2009) Biofilms of non-Candida albicans Candida species: quantification, structure and matrix composition. Med. Mycol. 47, 681-689
65 Mukherjee, P.K. and Chandra, J. (2004) Candida biofilm resistance. Drug Resist. Updat. 7, 301-309

66 Kumamoto, C.A. (2002) Candida biofilms. Curr. Opin. Microbiol. 5, $608-611$

67 Jain, N.et al. (2007) Biofilm formation by and antifungal susceptibility of Candida isolates from urine. Appl. Environ. Microbiol. 73, 1697-1703

68 Donlan, R.M. and Costerton, J.W. (2002) Biofilms: survival mechanisms of clinically relevant microorganisms. Clin. Microbiol. Rev. 15, 167-193

69 Kuhn, D.M. et al. (2002) Comparison of biofilms formed by Candida albicans and Candida parapsilosis on bioprosthetic surfaces. Infect. Immun. 70, 878-888

70 Iraqui, I. et al. (2005) The Yak1p kinase controls expression of adhesins and biofilm formation in Candida glabrata in a Sir4p-dependent pathway. Mol. Microbiol. 55, 1259-1271

71 Lattif, A.A. et al. (2010) Characterization of biofilms formed by Candida parapsilosis, C. metapsilosis, and C. orthopsilosis. Int. J. Med. Microbiol. 300, 265-270

72 Shin, J.H. et al. (2002) Biofilm production by isolates of Candida species recovered from nonneutropenic patients: comparison of bloodstream isolates with isolates from other sources. J. Clin. Microbiol. 40, 1244-1248

73 Baillie, G.S. and Douglas, L.J. (2000) Matrix polymers of Candida biofilms and their possible role in biofilm resistance to antifungal agents. J. Antimicrob. Chemother. 46, 397-403

74 Al-Fattani, M.A. and Douglas, L.J. (2006) Biofilm matrix of Candida albicans and Candida tropicalis: chemical composition and role in drug resistance. J. Med. Microbiol. 55, 999-1008

75 Gacser, A. et al. (2007) Targeted gene deletion in Candida parapsilosis demonstrates the role of secreted lipase in virulence. J. Clin. Invest. 117, 3049-3058 